\title{
Combined Extractive Dearomatization, Desulfurization, and Denitrogenation of Oil Fuels Using Deep Eutectic Solvents: A Parametric Study
}

\section{Supporting Information}

Ahmad S. Darwish*, Samah E. E. Warrag*, Farah O.S. Abuhatab, Idowu A. Adeyemi, Maaike C. Kroon, and Enas I. Nashef**

Khalifa University of Science and Technology, Center for Membrane and Advanced Water Technology

(CMAT), Chemical Engineering Department,

P.O. Box 54224, Abu Dhabi, United Arab Emirates (UAE)

*The authors have equally contributed to this work

**Corresponding Author: Enas M. Nashef, enas.nashef@,ku.ac.ae 


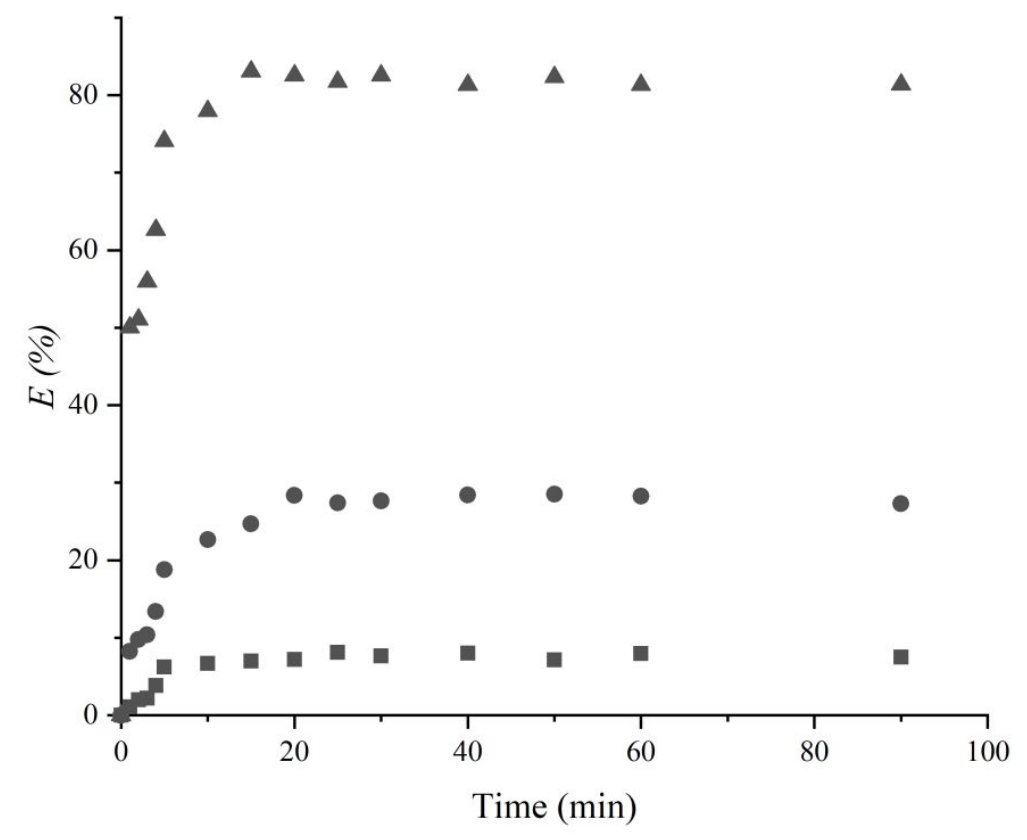

Figure S.1: Effect of stirring time on the extraction efficiency. Symbols: $(\boldsymbol{\Delta})$ E for quinoline, $(\bullet) \mathrm{E}$ for thiophene, and (घ) E for toluene. (Conditions: $S: F=1: 1, T=298.2 \mathrm{~K}, P=1.01$ bar; and settling time $=$ $1 h)$

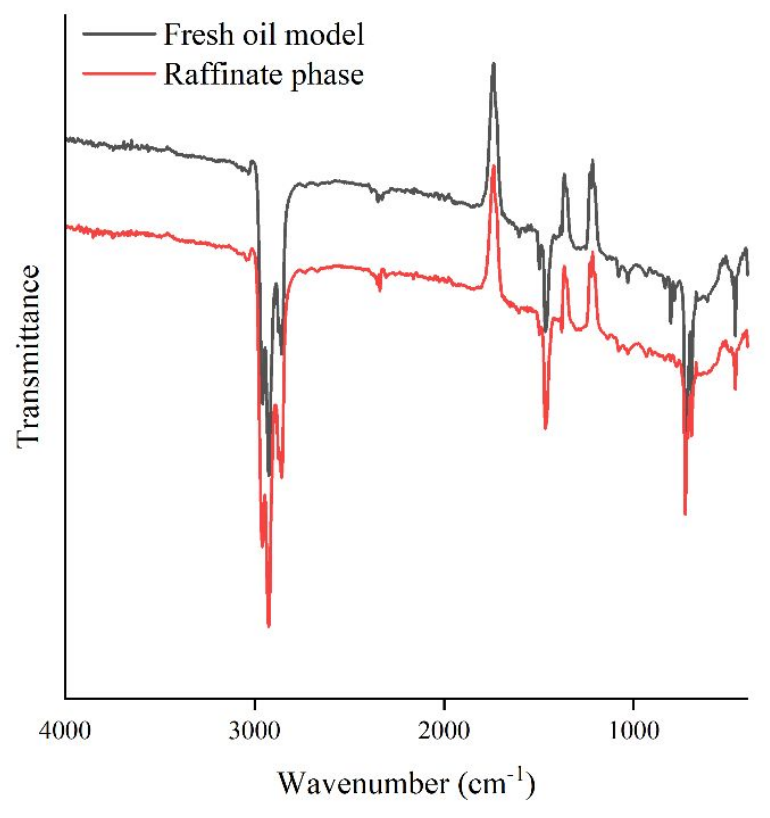

Figure S.2: FTIR analysis of a fresh oil mixture sample and an extracted oil mixture "raffinate" 
Table S.1: Numerical values of the DES "MTPPBr:TEG" density measured at a temperature range of $293.2 \mathrm{~K}-368.2 \mathrm{~K}^{\mathrm{a}}$

\begin{tabular}{|c|c|}
\hline Temperature $[\mathrm{K}]$ & Density $(\rho)\left[\mathrm{g} / \mathrm{cm}^{3}\right]$ \\
\hline 293.2 & 1.194 \\
\hline 298.2 & 1.190 \\
\hline 303.2 & 1.187 \\
\hline 308.2 & 1.183 \\
\hline 313.2 & 1.180 \\
\hline 318.2 & 1.176 \\
\hline 323.2 & 1.172 \\
\hline 328.2 & 1.169 \\
\hline 333.2 & 1.165 \\
\hline 338.2 & 1.162 \\
\hline 343.2 & 1.158 \\
\hline 348.2 & 1.154 \\
\hline 353.2 & 1.151 \\
\hline 358.2 & 1.147 \\
\hline 363.2 & 1.144 \\
\hline 368.2 & 1.140 \\
\hline
\end{tabular}

${ }^{\mathrm{a}}$ standard uncertainty in density $\mathrm{u}(\rho)= \pm 0.001$

Table S.2: Experimental LLE data in weight fractions for the pseudo-ternary systems $\{n$-heptane + thiophene + MTPPBr:TEG $\},\{n$-heptane + quinoline + MTPPBr:TEG $\}$, and $\{n$-heptane + toluene + MTPPBr:TEG $\}$ measured at $298.2 \mathrm{~K}$ and 1.01 Bar. $\boldsymbol{\beta}_{2}$ and $\boldsymbol{\beta}_{1}$ are the calculated distribution coefficient for the solute and the $n$-heptane, respectively. $\boldsymbol{S}$ are the selectivity values, $\boldsymbol{w}_{2, i}$ is the experimental initial composition of the solute, and $\boldsymbol{E}$ is the extraction efficiency per stage ${ }^{a}$

\begin{tabular}{|c|c|c|c|c|c|c|c|c|c|c|c|c|}
\hline \multirow[b]{2}{*}{$w_{2, i}$} & \multicolumn{4}{|c|}{$n$-alkane-rich phase } & \multicolumn{4}{|c|}{ DES-rich phase } & \multirow[b]{2}{*}{$\beta_{2}$} & \multirow[b]{2}{*}{$\beta_{1}$} & \multirow[b]{2}{*}{$S$} & \multirow[b]{2}{*}{$E$} \\
\hline & \multicolumn{2}{|c|}{$w_{1, R}$} & \multicolumn{2}{|c|}{$w_{2, R}$} & \multicolumn{2}{|c|}{$w_{l, E}$} & \multicolumn{2}{|c|}{$w_{2, E}$} & & & & \\
\hline \multicolumn{13}{|c|}{$n$-heptane (1) + thiophene (2) + MTPPBr:TEG (3) } \\
\hline$\underline{2 \%}$ & $\underline{0.986}$ & \pm 0.001 & $\underline{0.014}$ & \pm 0.001 & $\underline{0.004}$ & \pm 0.001 & $\underline{0.009}$ & \pm 0.001 & $\underline{0.647}$ & $\underline{0.004}$ & 161.4 & $\underline{32.7 \%}$ \\
\hline $10 \%$ & 0.936 & \pm 0.001 & 0.064 & \pm 0.001 & 0.005 & \pm 0.001 & 0.040 & \pm 0.001 & 0.622 & 0.005 & 126.2 & $36.8 \%$ \\
\hline $20 \%$ & 0.863 & \pm 0.001 & 0.137 & \pm 0.001 & 0.005 & \pm 0.001 & 0.074 & \pm 0.001 & 0.541 & 0.006 & 93.2 & $31.8 \%$ \\
\hline $30 \%$ & 0.796 & \pm 0.002 & 0.204 & \pm 0.002 & 0.006 & \pm 0.001 & 0.113 & \pm 0.001 & 0.556 & 0.007 & 74.2 & $31.9 \%$ \\
\hline $40 \%$ & 0.713 & \pm 0.006 & 0.287 & \pm 0.006 & 0.007 & \pm 0.001 & 0.143 & \pm 0.003 & 0.498 & 0.010 & 50.9 & $27.9 \%$ \\
\hline $50 \%$ & 0.631 & \pm 0.003 & 0.369 & \pm 0.003 & 0.007 & \pm 0.001 & 0.172 & \pm 0.002 & 0.467 & 0.011 & 43.4 & $26.1 \%$ \\
\hline $60 \%$ & 0.547 & \pm 0.001 & 0.453 & \pm 0.001 & 0.008 & \pm 0.001 & 0.218 & \pm 0.002 & 0.480 & 0.015 & 31.0 & $24.5 \%$ \\
\hline $70 \%$ & 0.419 & \pm 0.001 & 0.581 & \pm 0.001 & 0.008 & \pm 0.001 & 0.234 & \pm 0.005 & 0.402 & 0.019 & 20.8 & $16.7 \%$ \\
\hline \multicolumn{13}{|c|}{-heptane (1) + quinoline (2) + MTPPBr:TEG (3) } \\
\hline$\underline{2 \%}$ & $\underline{0.998}$ & \pm 0.001 & $\underline{0.002}$ & \pm 0.001 & $\underline{0.004}$ & \pm 0.001 & $\underline{0.020}$ & \pm 0.004 & $\underline{9.574}$ & $\underline{0.004}$ & $\underline{2327.9}$ & $\underline{89.9 \%}$ \\
\hline $10 \%$ & 0.987 & \pm 0.002 & 0.013 & \pm 0.001 & 0.005 & \pm 0.001 & 0.080 & \pm 0.001 & 6.180 & 0.005 & 1143.6 & $87.2 \%$ \\
\hline $20 \%$ & 0.972 & \pm 0.001 & 0.028 & \pm 0.001 & 0.006 & \pm 0.001 & 0.148 & \pm 0.003 & 5.297 & 0.006 & 823.0 & $86.1 \%$ \\
\hline $30 \%$ & 0.960 & \pm 0.004 & 0.040 & \pm 0.004 & 0.008 & \pm 0.001 & 0.213 & \pm 0.009 & 5.279 & 0.008 & 636.6 & $86.6 \%$ \\
\hline $40 \%$ & 0.945 & \pm 0.001 & 0.055 & \pm 0.001 & 0.010 & \pm 0.001 & 0.265 & \pm 0.009 & 4.770 & 0.010 & 460.5 & $86.1 \%$ \\
\hline
\end{tabular}




\begin{tabular}{|ccccccccccccc|}
$50 \%$ & 0.930 & \pm 0.001 & 0.070 & \pm 0.001 & 0.011 & \pm 0.001 & 0.315 & \pm 0.001 & 4.517 & 0.012 & 376.4 & $86.1 \%$ \\
$60 \%$ & 0.915 & \pm 0.002 & 0.085 & \pm 0.002 & 0.013 & \pm 0.001 & 0.357 & \pm 0.009 & 4.186 & 0.015 & 283.7 & $85.8 \%$ \\
$70 \%$ & 0.904 & \pm 0.001 & 0.096 & \pm 0.001 & 0.016 & \pm 0.001 & 0.396 & \pm 0.008 & 4.120 & 0.017 & 235.8 & $86.3 \%$ \\
& & & \multicolumn{7}{c}{ n-heptane (1) + toluene (2) } & MTPPBr:TEG (3)\} \\
$10 \%$ & 0.908 & \pm 0.001 & 0.092 & \pm 0.001 & 0.004 & \pm 0.001 & 0.011 & \pm 0.002 & 0.121 & 0.004 & 30.6 & $9.5 \%$ \\
$\underline{20 \%}$ & $\underline{0.814}$ & \pm 0.001 & $\underline{0.186}$ & \pm 0.001 & $\underline{0.003}$ & \pm 0.001 & $\underline{0.022}$ & $\underline{ \pm 0.001}$ & $\underline{0.116}$ & $\underline{0.004}$ & $\underline{28.5}$ & $\underline{7.5 \%}$ \\
$30 \%$ & 0.727 & \pm 0.002 & 0.273 & \pm 0.002 & 0.004 & \pm 0.001 & 0.034 & \pm 0.001 & 0.125 & 0.005 & 25.6 & $9.0 \%$ \\
$40 \%$ & 0.623 & \pm 0.003 & 0.377 & \pm 0.003 & 0.003 & \pm 0.001 & 0.043 & \pm 0.003 & 0.115 & 0.005 & 22.5 & $5.7 \%$ \\
$50 \%$ & 0.532 & \pm 0.002 & 0.468 & \pm 0.002 & 0.004 & \pm 0.001 & 0.056 & \pm 0.002 & 0.120 & 0.007 & 18.3 & $6.5 \%$ \\
$60 \%$ & 0.432 & \pm 0.008 & 0.568 & \pm 0.008 & 0.003 & \pm 0.001 & 0.065 & \pm 0.001 & 0.114 & 0.007 & 17.2 & $5.4 \%$ \\
$70 \%$ & 0.329 & \pm 0.002 & 0.671 & \pm 0.002 & 0.003 & \pm 0.001 & 0.088 & \pm 0.001 & 0.131 & 0.008 & 15.7 & $3.9 \%$ \\
$80 \%$ & 0.227 & \pm 0.003 & 0.773 & \pm 0.003 & 0.002 & \pm 0.001 & 0.091 & \pm 0.001 & 0.118 & 0.010 & 12.1 & $3.2 \%$ \\
\hline
\end{tabular}

${ }^{a}$ The DES (in mole fraction) consists of methyltriphenylphosphonium bromide $\left(\mathrm{x}_{\mathrm{MTPPBr}}=0.1927\right)$, triethyleneglycol $\left(\mathrm{x}_{\mathrm{TEG}}=0.7707\right)$, and water $\left(\mathrm{x}_{\mathrm{H} 2 \mathrm{O}}=0.0365\right)$. The DES composition $\left(\mathrm{w}_{3}\right)$ in both phased can be calculated via mass balance. Standard uncertainty in temperature $u(\mathrm{~T})= \pm 0.1, u(\mathrm{p})=0.04$ bar, and for the DES (in mole fraction) $u\left(\mathrm{x}_{\mathrm{TEG}}\right)$ $=\mathrm{u}\left(\mathrm{x}_{\mathrm{MTTPBr}}\right)=\mathrm{u}\left(\mathrm{x}_{\mathrm{H} 2 \mathrm{O}}\right)=0.0002$.

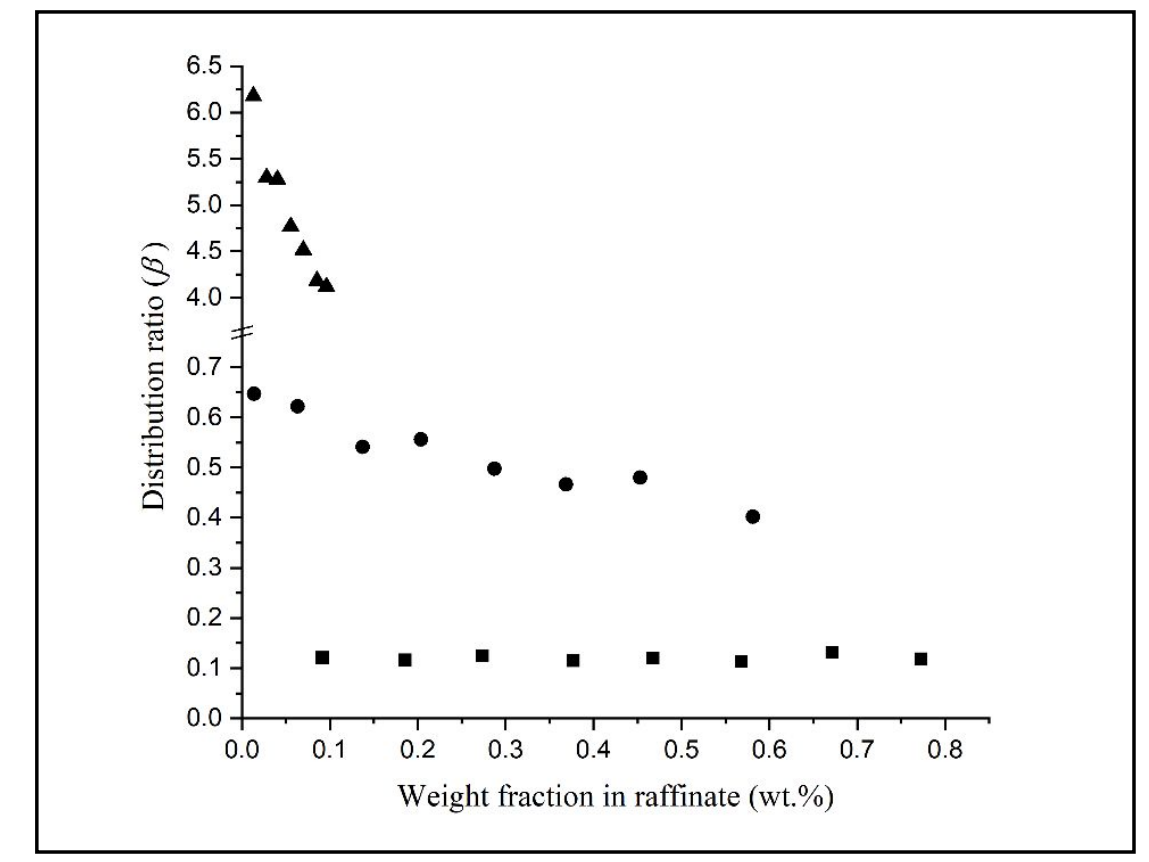

Figure S.3: Solute distribution coefficient as a function of the weight fraction of oil impurity in the $n$ alkane-rich phase. The weight fractions are shown as $(\boldsymbol{\Delta})$ for quinoline, $(\bullet)$ for thiophene, and $(\boldsymbol{\square})$ for toluene 


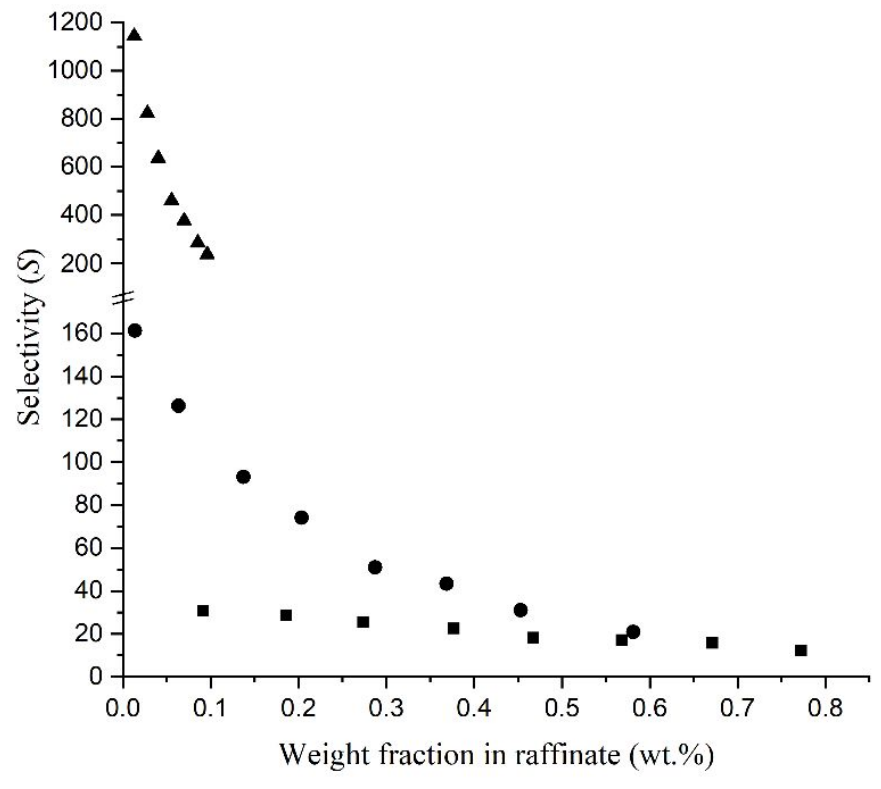

Figure S.4: Selectivity as a function of the weight fraction of oil impurity in the $n$-alkane-rich phase. The weight fractions are shown as $(\boldsymbol{\Delta})$ for quinoline, $(\bullet)$ for thiophene, and $(\boldsymbol{\bullet})$ for toluene

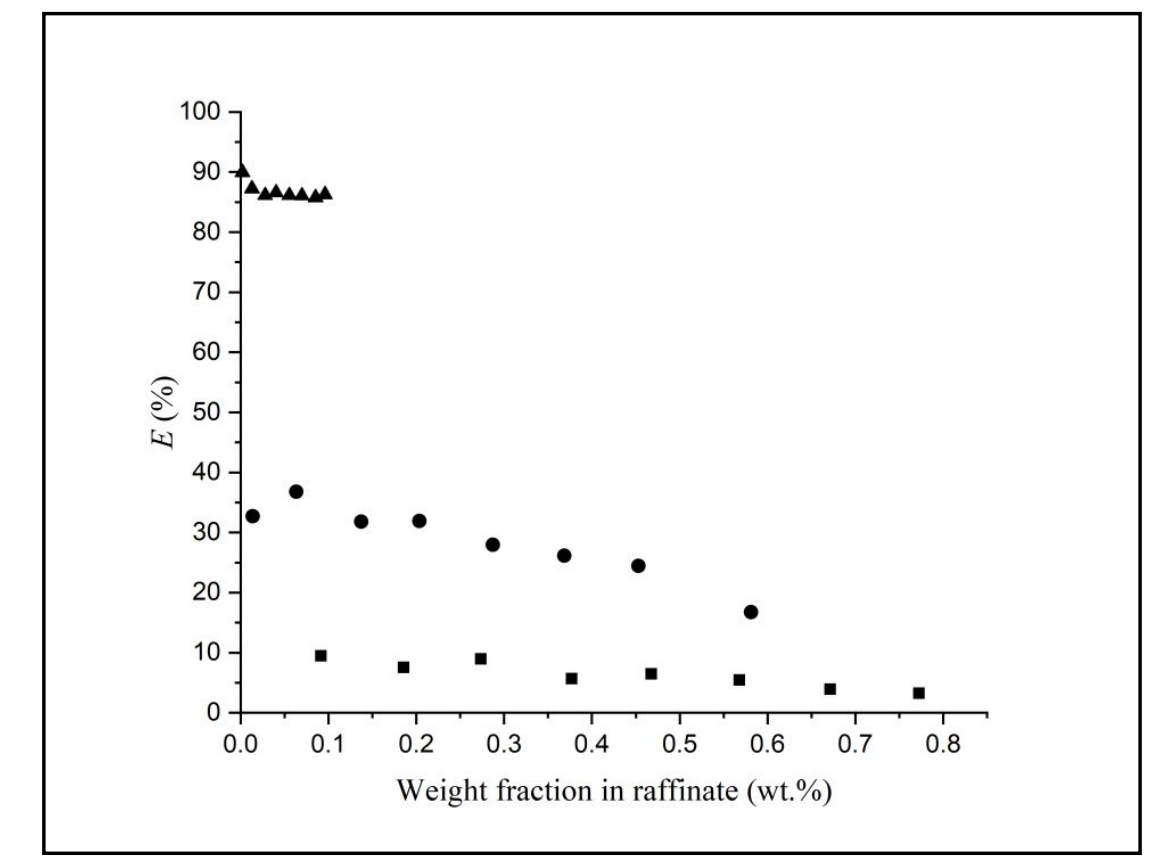

Figure S.5: Extraction efficiency per tie-line as a function of the weight fraction of oil impurity in the nalkane-rich phase. The weight fractions are shown as $(\boldsymbol{\Delta})$ for quinoline, $(\bullet)$ for thiophene, and $(\boldsymbol{\square})$ for toluene 